\title{
Namenregister / Index of Names
}

Abramson, Jill 66

Acosta, Jim 215

Adenauer, Konrad 170

Adorno, Theodor W. 29, 83, 181, 187

Aesop 47

Alvarez, Julia $282 f$.

Amis, Martin 54

Andersen, Hans Christian 61

Anderson, Benedict 85, 301

Anderson, Sherwood 231

Anthias, Floya 290

Arendt, Hannah 23, 90

Aristoteles 1, 39

Armstrong, Nancy 220

Ashe, Stephen 302

Asher, Evan Joseph $72 \mathrm{f}$.

Assmann, Aleida 107, 165, 172

Assmann, Jan 107, 165

Atwood, Margaret 54, 227, 229

Austen, Jane $224 \mathrm{f}$.

Auster, Paul 48

Avery, Dylan 128

Badcock, John 248

Baez, Joan 61

Baier, Christian 141, 217

Bakhtin, Mikhail 220

Ball, James 298

Bannon, Steve 284, 292

Barkun, Michael 133

Barthes, Roland 281, 285

Becker, Frank 163

Bee, John 248

Behn, Aphra 222

Benhabib, Seyla 71

Benjamin, Walter 142, 219

Benko, Ralph $74 \mathrm{f}$.

Bennington, Geoff 216

Berger, John 310

Bergerac, Cyrano de 248

Biden, Hunter 56
Biden, Joe 56, 62

bin Laden, Osama $52 \mathrm{f}$.

Blödorn, Andreas 168

Bloom, Leopold 227

Blumenberg, Hans 6, 157, 165, 173, 219, 222, 230

Boltanski, Luc $307 f$.

Bolton, John 58

Boxall, Peter 263

Bradbury, Malcolm 310

Braddon, Mary Elizabeth 226

Bragg, Billy 313

Brandt, Willy 170

Breithaupt, Fritz 36, 265, 267f., 270

Breitinger, Johann Jakob $32 \mathrm{f}$.

Breslow, Jason M. 50, 52

Brontë, Charlotte 225

Browning, Gary K. 70f.

Brueggemann, Walter 218

Bude, Heinz 89

Burney, Frances $224 \mathrm{f}$.

Bush, George W. $49 \mathrm{ff} ., 131$

Butter, Michael 38, 131, 133, 140, 149f., 152

Bystron, Petr 203

Camus, Renaud 39

Cantiello, Jessica 282

Carlson, Eric 248

Carlyle, Thomas 250

Cartwright, Anthony 312

Cassirer, Ernst 157f., 160

Castagnaro, Mario 235, 246

Castillo, Ana 282

Chabe, Tamara 305

Chin, Frank 289

Churchill, Winston 309

Cillizza, Chris 60

Clancy, Tom 153

Clinton, Bill 50, 55ff., 61

Clinton, Hillary 25, 37

Coe, Jonathan 297f., 306ff., 312, $316 f$.

¿ Open Access. (C) 2021 Antonius Weixler, Matei Chihaia, Matías Martínez, Katharina Rennhak, Michael Scheffel und Roy Sommer, publiziert von De Gruyter. (c) BY Dieses Werk ist lizenziert unter der Creative Commons Attribution 4.0 International Lizenz. 
Cohen, Lara Langer 234

Cohn, Roy 57

Collins, Wilkie 226

Colm Hogan, Patrick 274

Conrad, Joseph 226

Conway, Kellyanne 66

Craig, Amanda 312

Crouch, Colin 61

Crow, Jason 58

Crusoe, Robinson 225

Cruz, Angie 282

Cubitt, Geoffrey $133 f$.

Cullen, Darren 117

Cumberbatch, Benedict 299

Cummings, Dominic 299

Cusk, Rachel 231

Cutter, Martha 289

D’Ancona, Matthew 14, 66, 73, 298

Dağdelen, Sevim 203

Dalloway, Clarissa 227

Danto, Arthur C. 16

Davies, William 75

Davy, Sir Humphrey 252

Dedalus, Stephen 227

Defoe, Daniel $224 \mathrm{f}$.

Deighton, Len 308

Demeyer, Hans $263 \mathrm{f}$.

DeParle, Jason 74

Derrida, Jacques 288

Díaz, Junot 282

Dickens, Charles 266, 314

Diehl, Paula 160

Dinius, Marcy 243, 255

Dix, Andrew 290

Doll, Martin 235

Donner, Richard 153

Duffy, Carol Ann 312

Dumm, Sebastian 159

Dunning, David 151

Durkheim, Émile 181

Dyk, Silke van 24, 27

Eaglestone, Robert 306

Edgar, David 310

Edwards, John Clarke 238

Elisabeth II. 305
Elsässer, Jürgen 203

Emerson, John B. 203

Erdrich, Louise 289

Erhard, Ludwig 167, 170

Esposito, Elena 106, 223

Evans, Alex 216ff., 224

Evaristo, Bernardine 306

Farage, Nigel 67, 299ff., 315

Fassbinder, Rainer Werner $169 \mathrm{f}$.

Faulkner, William 286

Feinstein, Dianne 53

Fleming, Ian 308

Fleuß, Dannica 159

Floyd, George 54

Fluck, Winfried 292

Flynn, Michael 56

Foroughi, Hamid 217

Foster, Hal 263

Frankfurt, Harry G. 27f., 91

Fraser, Nancy $229 f$.

Frick, Werner 150

Fries, Stefan 131

Funk, Wolfgang 288

Fuqua, Antoine 153

Gabriel, Sigmar 138

Gadinger, Frank 36

García, Yolanda 282

Gardner, Cory 58

Gaskell, Elizabeth 228, 231

Gates, Bill 128, 133, 148

Gauland, Alexander 203

Geertz, Clifford 276

Gerassimow, Walerij 202

Gibbs, Laura 47

Giles, Peter $220 f$.

Gilroy, Paul $304 \mathrm{ff}$.

Giuliani, Rudy 56f., 60

Gladwell, Malcolm 54

Goethe, Johann Wolfgang von $224 \mathrm{f}$.

Goodman, Sam $307 f$.

Göttlich, Udo 191

Gove, Michael 299

Gramsci, Antonio 230

Greenwald Smith, Rachel 263 
Greig, David 310

Groh, Dieter 152

Habermas, Jürgen 6, 177, 180ff., 192, 194

Halbwachs, Maurice 165

Hale, Margaret 228

Hardy, Thomas 226

Hari, Mata 170

Hartmann, Nicolai 86

Haselstein, Ursula 288

Hassknecht, Gerold 187

Haynes, Toby 312

Heins, Volker 193

Hempel, Carl 17

Hendricks, Vincent F. 13f., 25, 31, 116

Hepfer, Karl 31

Herman, Eva 138

Hermand, Jost 168

Herschel, Sir John 242, 252

Hertmans, Stefan 264f., $272 \mathrm{ff}$., 277

Hickenlooper, John 58

Higgins, Nicholas 228

Hildmann, Attila 128, 139

Hilzinger, Sonja 35

Hochschild, Arlie Russell 4, 9, 65, 72, 74ff., 119, 281, $292 \mathrm{f}$.

Höcke, Björn $137 f$.

Hoffmann, Kurt 168

Hölscher, Lucien 85

Horkheimer, Max 181, 187

Horn, Eva 28

Huck, Christian 136, 139ff., 144

Hugonnier, François 48

Humboldt, Alexander 252

Huntington, Samuel 284

Hussein, Saddam 49, 51 .

Hythloday, Raphael $221 f$.

Imhoff, Roland 135

Irving, Washington 248

James, Henry 226

Jarvis, Brian 290

Jebsen, Ken 203

Jen, Gish 293

Jenkins, Henry 5, 103, 105, 113f., 117

Jenner, Paul 290
Johnson, Boris 316

Joyce, James 227

Kavanagh, Joseph 316

Kay, Jackie 228

Keen, Suzanne 266, 270f., 275

Kessler, Glenn 57

Kiesinger, Hans Georg 170

Kingston, Maxine Hong 289

Klein, Christian 33, 38

Kluge, Alexander 179

Kohl, Helmut 174

Koppetsch, Cornelia 89

Kornelius, Stefan 216

Koschorke, Albrecht 28, 34, 36, 109, 118, 147f., 151, 173

Kosofsky Sedgwick, Eve 226

Kracauer, Siegfried 162, 166

Krämer, Lucia 288

Kretschmer, Michael 93f.

Kristeva, Julia 109

Kruger, Justin 151

Kuttner, Robert 284

Lahiri, Jhumpa 8, 282f., 293

Lanchester, John 231, 312f., 316

Langenohl, Andreas 304

Langer, Bernhard 37

Laplace, Pierre 252

Laschet, Armin 199

le Carré, John 308

Le Pen, Marine 106

Le, Nam 8, 286

Leclercq, Christophe 204

Lee, Gus 293

Lehmann, Johannes 32

Leonhardt, David 53

Lessenich, Stephan 88

Lethen, Helmut $144 \mathrm{f}$.

Leucht, Robert 110

Lewinsky, Monika 50

Lewis, Clive Staples 219

Lindner, Rolf 181

Lobo, Sascha 173

Locke, Richard Adams 236, 242, 255

Lotman, Jurij M. 5, 103, 105, 107ff., 114 
Lozada, Carlos $74 \mathrm{f}$.

Luhmann, Niklas 29, 134, 144, 180

Lukács, Georg 219

Lyotard, Jean-François 4, 65, 67ff., 76, 78ff., 216

Mackie, John L. 17

Mahjoub, Jamal 306

Mahler, Andreas 109ff., 114

Maizière, Thomas de 201

Mance, Henry 299

Martinez, Carlo 246, 249, 255

Martínez, Matías 33, 38, 149, 301

Massumi, Brian 216

Matychak, Tetiana 203

Maus, Heinz 96

May, Theresa $300 \mathrm{ff} ., 315$

McCarthy, Jenny $72 f$.

McConnell, Mitch 55, $58 \mathrm{f}$.

McEwan, Ian 297f., 303ff., 312, 317

McHale, Brian 264

McKeon, Michael 220, 224

Menasse, Robert 84

Merkel, Angela 148

Merz, Friedrich 205

Michaels, Walter Benn 291

Mishra, Pankaj 226, 229

Mitford, Mary Russell 230

Monck Mason, Thomas 252

More, Thomas $220 \mathrm{ff}$.

Morton, John 221

Moser, Christian 35

Moynihan, Daniel Patrick 85

Mueller, Robert 56

Müller, Michael 34, 36

Müller, Wolfgang 168

Müller-Funk, Wolfgang 80

Mulvaney, Mick $57 \mathrm{f}$.

Münkler, Herfried 161, 165, 167, 174

Musab al-Zarqawi, Abu 52

Mutsaers, Charlotte 265, 269, 276

Nagel, Thomas 276

Naidoo, Xavier 139

Negt, Oskar 179

Neiman, Susan 78

Neubauer, Hans-Joachim 29
Neuss, Wolfgang 168

Niekerk, Carl 110

Niketas, Kyrios 65

Noyce, Philip 153

Obama, Barack 4, 53, 57, 66f., 74, 77f., 81, 293

Ochsenreiter, Manuel 203

Park, Robert Ezra 181

Parks, Tim 310

Passerini, Luisa 310

Paul, Robert William 161

Peeters, Koen 264f., 269ff., 275

Pelosi, Nancy 58

Pfahl-Traughber, Armin 131

Pfeijffer, Ilja Leonard 265

Philippe, Louis 96

Phillips, Caryl 306

Phillips, Mike 306

Pinker, Stephen 52

Pittel, Harald 316

Poe, Edgar Allan 8, 233, 235, 237, 241, $245 \mathrm{ff}$.

Pollin, Burton 256

Popper, Karl 131

Pörksen, Bernhard 105, 159

Powell, Colin 4, 47, 49ff., 132

Prometheus 47f.

Propp, Wladimir 292

Putin, Wladimir 57, 202

Quendler, Christian 249

Reckwitz, Andreas 219

Reinfandt, Christoph 223, 229, 313

Richardson, Samuel $224 \mathrm{f}$.

Ricœur, Paul 76

Riesman, David 181

Roberts, David 94

Robertson, Pat 133

Rooney, Sally 228

Rorty, Richard 86

Rostek, Joanna 313

Roth, Philip 227

Rowling, J. K. 54

Rubio, Marco 49, $59 f$.

Rushdie, Salman 227 
Ryan, Donal 231

Ryan, Marie-Laure 58

Said, Edward 95

Sanders, Bernie 293

Sarasin, Phillip 24

Sayer, Derek 298, 302

Schaal, Gary S. 159

Scheffel, Michael 149

Schmidt, Helmut 170

Schmitt, Carl 86f.

Schröder, Gerhard 171

Schudson, Michael 234

Sebald, W. G. 268

Seehofer, Horst 107

Seemann, Michael 173

Seeßlen, Georg 107

Seidler, Victor J. 298

Shakespeare, William 48

Shaw, Kristian 312

Shelley, Mary 226

Shiller, Robert J. 217

Siegumfeldt, I. B. 48

Silko, Leslie Marmon 289

Smith, Ali 312, 314

Smith, Sidonie 286

Smith, Zadie 314

Snyder, Timothy 216

Söder, Markus 5, 103f., 106ff.

Sollors, Werner 286

Sommer, Roy 275, 313

Sondland, Gordon 57

Sparhawk, Edward Vernon 255

Spencer, Herbert 181

Spicer, Sean 66

Spiering, Menno 299, 302

Spivak, Gayatri Ch. 95

Stein, Lorenz von 97

Sternheim, Carl 98

Storch, Beatrix von 203

Sturt, George 231

Tan, Amy 282, 293

Taylor, Charles 287

Tew, Philip 263

Thornton, John 228
Tocqueville, Alexis de 181, 192

Todd, Chuck 56, 66

Todorov, Tzvetan 55

Tremain, Rose 228

Trilling, Lionel 287

Trollope, Anthony 228

Trump, Donald 5, 14, 23f., 37ff., 47ff., 53ff., 65f., 78, 85, 91, 106, 127f., 130, 135, 137, 140, 144, 178, 203, 215, 293, 298

Trump, Mary 53

Tucker, George 248

Tückmantel, Ulrich 132

Turner, Victor 313, 315

Tyler, Anne 282

Ulfkotte, Udo 203

Vestergaard, Mads 13f., 25, 31, 116

Vitse, Sven $263 \mathrm{f}$.

Wachowski, Lana 152

Wachowski, Lilly 152

Walsh, Lynda 234, 252

Walsh, Richard 266

Warren, Septimus 227

Watson, Julia 286

Watt, Ian 220

Weber, Max 158, 183, 193

Wells, H. G. 161

Werner, Welf 292

White, Hayden 19, 129

Wilders, Geert 106

Wineburg, Sam 208

Winfrey, Oprah $72 f$.

Wippermann, Wolfgang 131

Wittgenstein, Ludwig 69,71

Wood, Ellen 226

Woolf, Virginia $227 \mathrm{f}$.

Wortmann, Sönke 171

Zelensky, Volodymyr 57

Ziervogel, Maike 315

Zuckerman, Ethan 172

Zwierlein, Anne-Julia 313 
